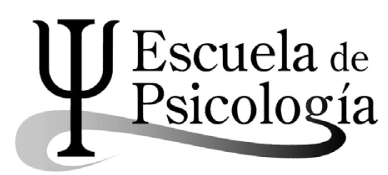

Wímb lu, Rev. electrónica de estudiantes Esc. de psicología, Univ. de Costa Rica. 9 (2): 7-21, 2014 / ISSN: 1659-2107

\title{
EFECTO DEL ASCO SOBRE LAS ACTITUDES HACIA HOMBRES HOMOSEXUALES: \\ EVIDENCIAS DE COGNICIÓN CORPORALIZADA EN UNA MUESTRA COSTARRICENSE ${ }^{1}$
}

\author{
Effect of disgust on attitudes toward gay men: \\ Evidence of an embodied cognition in a Costa Rican sample
}

Julianne Beirute-Herrera*

Valeria Angulo-Castro**

Karina Fallas-Gamboa***

Jorge Esteban Prado-Calderón****

\begin{abstract}
Resumen: Las teorías sobre cognición corporalizada plantean que los estados internos del cuerpo juegan un rol fundamental en los procesos cognitivos. Los estados corporales pueden modular incluso las actitudes hacia grupos sociales. En esta investigación se examinó cómo la reacción emocional de asco influye sobre las actitudes hacia varios grupos sociales, en particular hacia los hombres homosexuales. Para ello se pidió a los participantes del grupo experimental $(n=23)$ contestar un cuestionario mientras se encontraban en un aula con un olor ambiental desagradable. El cuestionario incluyó un termómetro de sentimientos para estimar las actitudes hacia diversos grupos sociales y una escala para evaluar las actitudes hacia específicamente personas homosexuales. Se encontró que en el primer instrumento las personas expuestas al mal olor ambiental reportaron actitudes más negativas hacia varios grupos sociales que las personas del grupo control $(n=18)$, especialmente hacia los hombres homosexuales $(d=1.01)$. Sin embargo en el segundo instrumento, aunque la tendencia estuvo presente, los resultados no fueron estadísticamente significativos. En síntesis, se observaron evidencias del efecto del estado corporal de asco sobre las actitudes hacia varios grupos sociales, pero la evidencia acerca del efecto específico sobre las actitudes hacia hombres homosexuales no fue consistente.
\end{abstract}

Palabras clave: asco, emoción, homofobia, discriminación, cognición corporalizada.

\footnotetext{
* Universidad de Costa Rica, Escuela de psicología, Costa Rica. Correo: beirutej@gmail.com

** Universidad de Costa Rica, Escuela de psicología, Costa Rica. Correo: vale.angulo22@gmail. com

*** Universidad de Costa Rica, Escuela de psicología, Costa Rica. Correo: kari-fallas@hotmail.com

**** Universidad de Costa Rica, Escuela de psicología, Costa Rica. Correo: jorgepradopsi@ gmail.com

Recepción: 23/5/2013 Aceptación: 30/10/2013
} 


\begin{abstract}
Embodied cognition theories suggest that the internal states of the body play a crucial role in cognitive processes. Body states can modulate even attitudes toward social groups. This research examined how the emotional reaction of disgust influences on attitudes toward various social groups, particularly toward gay men. For this, the participants in the experimental group $(n=23)$ were asked to complete a questionnaire in a classroom with an unpleasant ambient odor. The questionnaire included a feelings thermometer to estimate attitudes toward various social groups and a scale to assess attitudes toward homosexuals specifically. We found that in the first instrument people exposed to unpleasant ambient odor reported more negative attitudes toward various social groups that people in the control group $(n=18)$, especially toward gay men ( $d=$ 1.01). However in the second instrument, although the trend was present, the results were not statistically significant. In summary, evidence of the effect of body state of disgust on attitudes toward various social groups were observed, but evidence about the specific effect on attitudes toward gay men was not consistent.
\end{abstract}

Key Words: disgust, emotion, homophobia, discrimination, embodied cognition.

\title{
Introducción
}

La teoría sobre embodiment indica que la posición corporal o del rostro, los cambios o movimientos, y los correlatos internos como la activación fisiológica - la dificultad para respirar pueden cambiar la formación y el mantenimiento de diversas cogniciones, entre ellas las actitudes, ya que influyen en la manera en que las personas procesan la información (Schnall, Haidt, Clore \& Jordan, 2008). Tal como lo señalan Lakoff y Johnson (1999), los efectos del embodiment sobre las actitudes se manifiestan en la medida en que los individuos cambian su manera de evaluar objetos, personas y demás estímulos según sus estados corporales internos. Acorde con estos autores, el embodiment incluye aspectos como las posiciones de la cara, los movimientos del cuerpo y movimiento de acercamiento o evitación.

Los estudios sobre el tema de embodiment ganaron relevancia en la década de 1960, por ejemplo Solarz (1960) descubrió que las personas son más rápidas al halar una palanca hacia ellos cuando ven una palabra positiva que cuando esta es negativa. Bem (1972) y Laird (1974) encontraron que los individuos experimentan estados afectivos que guardaron relación con posturas específicas. Mientras que Wells y Petty (1980) comprobaron que las personas que asentaban con la cabeza al escuchar un mensaje estaban mayormente predispuestas a estar de acuerdo con dicho enunciado que las personas que no lo hicieron.

Inbar, Pizarro y Bloom (2012) han señalado también como embodiment la influencia de estímulos olfativos en la evaluación moral o la concepción del objeto o individuo en un momento determinado, ya que un olor desagradable suscita la emoción de asco, lo cual acarrea respuestas corporales innatas y expresiones faciales específicas. De esta forma sucede que el estado corporal afecta las actitudes o los procesos cognitivos de las personas, lo cual se conoce como embodied cognition o cognición corporalizada, ya que las emociones poseen cierta representación sensorial específica (Leung, Qiu, Ong \& Tam, 2011). 
En la presente investigación se buscó estimar mediante un aroma si la respuesta emocional de asco influye sobre las actitudes hacia varios grupos sociales, con el objetivo explícito de estimar el efecto del estado fisiológico de asco sobre las actitudes hacia los hombres homosexuales, tal y como se encontró previamente en Inbar et al. (2012). De esta forma se pretende aportar información a un tema relevante en la actualidad y de gran aporte social. Para esto se implementó un cuestionario a dos grupos, el grupo control y el experimental. El primero contestó el cuestionario sin que los investigadores manipularan el ambiente en el que se encontraban, mientras que el grupo experimental contestó el cuestionario con un olor desagradable disperso en el ambiente. Dicho cuestionario evaluó las actitudes de los individuos sobre diversos grupos y hacia las personas homosexuales.

De acuerdo con la bibliografía revisada para la realización de este estudio, la investigación sobre embodiment como teoría es relativamente reciente (Meier, Schnall, Schwarz \& Bargh, 2012). Específicamente en el contexto costarricense, existe un vacío en cuanto a la investigación en el tema, ya que toda la bibliografía consultada correspondía a autores extranjeros. Por esta razón planteamos la necesidad de investigar sobre la cognición corporalizada y su efecto; en este caso específico, la actitud hacia la población homosexual en un contexto costarricense, ya que al ser un estado confesional, todavía se perpetúa la homofobia entre la población, viéndose obstaculizado el respeto de los derechos de las personas homosexuales.

Tal y como lo exponen Smith-Castro y Molina-Delgado (2011), la homofobia comprende un conjunto de actitudes negativas hacia la conducta homosexual, hacia las personas homosexuales y hacia la comunidad LGBT (lesbianas, gais, bisexuales y transexuales) en general; por su parte, Moral (2013) la caracteriza como un terror irracional a ser homosexual y a tener contacto con personas homosexuales. La homofobia es un problema actual en el contexto latinoamericano y costarricense. Por ejemplo, Moral (2013) analizó los datos de una muestra probabilística de la población mexicana $(n=4383)$ y encontró que el $57 \%$ no aceptaría que una persona homosexual viviera en su casa, mientras que un $62 \%$ no aceptaría a un hijo homosexual. En el caso de Costa Rica, varios estudios evidencian que la homofobia es un problema actual. Suárez y Madrigal (2007) encuestaron a 500 jóvenes universitarios y reportaron que un $11 \%$ manifestó desagrado y disgusto hacia la homosexualidad o el lesbianismo. Fournier (2008) consultó a una muestra de 400 universitarios y encontró, por ejemplo, que altos porcentajes consideraban "chocante" ver a parejas besándose cuando estas estaban compuestas por personas homosexuales, ya fueran hombres (39.6\%) o mujeres (30.7\%). Asimismo, Smith-Castro y Molina-Delgado (2011) encuestaron a 100 universitarios heterosexuales y encontraron que grandes porcentajes se manifestaron en contra del matrimonio (48\%) y la unión civil $(37 \%)$ entre 
personas del mismo sexo. De manera coincidente, tanto el estudio mexicano de Moral (2013) como el costarricense de Smith-Castro y Molina-Delgado (2011) encontraron que la homofobia era mayor en el caso de los hombres y de las personas con creencias religiosas más ortodoxas.

En la presente investigación se toma en cuenta esta realidad. Sin embargo, el objetivo de la misma no es explorar ni determinar los niveles de homofobia en determinada población sino más bien estimar el efecto del estado fisiológico (en este caso, el asco) sobre las actitudes hacia determinados grupos sociales (en particular, hacia las personas homosexuales) como una evidencia experimental de cognición corporalizada.

\section{Método}

\section{Tipo de Investigación}

Con base en la literatura metodológica (Balluerka \& Vergara, 2002; Hernández, Fernández \& Baptista, 2010; Shadish, Cook \& Campbell, 2002) y el antecedente directo del presente estudio (Inbar et al., 2012) se utilizó un diseño experimental de tipo unifactorial e intersujeto. Como lo exponen Balluerka y Vergara (2002), es un diseño simple o unifactorial en tanto consta de una sola variable independiente, en este caso, la presencia o ausencia del olor desagradable; por otra parte, es un diseño intersujetos o intergrupos en tanto cada grupo recibe solo una de las manipulaciones, no las dos. En otras palabras, cada participante fue asignado a una sola condición experimental, a la de olor desagradable o a la condición sin olor, luego de lo cual se le consultó sobre sus actitudes hacia grupos sociales.

\section{Participantes}

La muestra consistió en 41 estudiantes de dos grupos de psicología de la Universidad de Costa Rica, 33 mujeres (80.5\%) y 8 hombres (19.5\%), con una edad promedio de 19.12 años. Fueron en su mayoría personas de orientación heterosexual $(85.4 \%)$, en soltería $(100 \%)$ y residentes de la provincia de San José $(65.9 \%)$. Del total de la muestra, 18 personas estuvieron en el grupo control siendo un $43.9 \%$, mientras que 23 personas respondieron el cuestionario en el grupo experimental, lo que corresponde a un $56.1 \%$ de la muestra.

\section{Instrumentos}

Se utilizó un cuestionario que incluyó las preguntas sobre datos sociodemográficos, las estimaciones de las actitudes hacia grupos sociales 
y personas homosexuales, y los ítems de control de variables extrañas y de comprobación de la manipulación.

En la primera sección, destinada a la información sociodemográfica, se consultó por el sexo, la edad, el estado civil, el domicilio permanente y la orientación sexual de las personas participantes. Seguidamente, en la segunda sección, se incluyeron dos estimaciones de las actitudes hacia grupos sociales: una hacia 15 grupos sociales, y otra orientada hacia personas homosexuales.

El primer instrumento para estimar actitudes consistió en una réplica del utilizado por Inbar et al. (2012) y descrito como "termómetro de sentimientos" (feeling thermometer). En este instrumento la persona participante debe indicar los sentimientos que le generan varios grupos sociales, para lo cual debe guiarse por un "termómetro" de sentimientos que va desde "muy frío o sentimiento muy desfavorable" (0) hasta "muy caliente o sentimiento muy favorable". Para cada grupo, la persona participante debe anotar un número del 0 al 100. De esta manera, mayores puntuaciones indican actitudes más positivas hacia el grupo social. Utilizando este instrumento, las personas participantes indicaron sus actitudes hacia los "hombres homosexuales", "mujeres homosexuales", "hombres heterosexuales" y "mujeres heterosexuales", así como hacia otros 11 grupos sociales (personas indígenas, chinas, colombianas, ateas, obesas, pobres, campesinas, adultas mayores, católicas, nicaragüenses y afrocostarricenses). Estos 11 grupos sociales adicionales fueron tomados de investigaciones interculturales recientes sobre estereotipos en las que se han utilizado muestras costarricenses (Cuddy et al., 2009; Durante et al., 2013), y fueron incluidos con el objetivo de dificultar que las personas participantes descubrieran que la hipótesis del estudio se refería solamente a las actitudes hacia personas homosexuales. Los 15 grupos fueron presentados en una misma secuencia aleatoria para todas las personas participantes.

El segundo instrumento fue específico para estimar actitudes hacia personas homosexuales. Se utilizó la versión en español (Cardenas \& Barrientos, 2008; Barrientos \& Cárdenas, 2012) de la escala Attitudes Toward Lesbians and Gay Men (ATLG, Herek, 1988). Este es el instrumento más comúnmente utilizado para estimar el prejuicio hacia personas homosexuales. Desarrollado por Herek, fue adaptado al español por este mismo autor y aplicado en ambos idiomas a una muestra de 616 descendientes de personas mexicanas en los Estados Unidos (Herek \& González-Rivera, 2006). Posteriormente, el instrumento fue probado en el contexto chileno por Cárdenas y Barrientos (2008) quienes encontraron adecuados niveles de consistencia interna (ATLG $=.94$, ATL $=.88$, ATG $=.91$ ) al aplicarlo a estudiantes universitarios ( $n=142)$. Asimismo, Barrientos y Cárdenas (2012) analizaron la estructura factorial del instrumento con 504 estudiantes chilenos y obtuvieron resultados adecuados. En la presente investigación también se obtuvieron índices de consistencia interna elevados con estudiantes 
universitarios costarricenses (ATLG $=.92, \mathrm{ATL}=.82, \mathrm{ATG}=.87$ ). La escala ATLG consiste en una escala de 20 ítems compuesta de dos subescalas, una de actitudes hacia lesbianas (ATL), y otra de actitudes hacia gays (ATG). Para cada ítem, la persona participante debe indicar su grado de acuerdo mediante una escala Likert de 5 puntos de "muy en desacuerdo" a "muy de acuerdo". Un ejemplo de ítem de la ATG es "Si un hombre tiene sentimientos homosexuales, debería hacer todo lo posible para superarlos", mientras que un ejemplo de ítem de la ATL es "Las lesbianas son enfermas". Luego de recodificados los ítems, se calcula su promedio, donde mayores puntuaciones indican mayor prejuicio, por lo que bajas puntuaciones indican actitudes favorables hacia las personas homosexuales.

Finalmente, el cuestionario incluyó una pregunta en la cual se consultaba al participante cuál consideraba que era la hipótesis que se sometía a prueba en el estudio. Además, para asegurar que la manipulación (generar asco) se diera según fue planificada, se indagó si el participante tenía disminuida su capacidad olfativa, si tenía un resfrío, si consideraba que hubo algún factor ambiental que interviniera en sus respuestas, cuál era este factor, qué tan desagradable e incómodo le resultó y, por último, se le consultó si sospechó que el olor era parte del experimento.

Para la manipulación experimental, el olor desagradable fue producido mediante un spray comercial con aromar a sudor llamado "Stinky Sweat Spray", el cual fue rociado en el aula previo al ingreso del grupo experimental. El aula correspondiente al grupo control no presentaba este olor desagradable.

\section{Procedimiento}

El experimento se llevó a cabo en aulas de clase de la Universidad de Costa Rica y fue presentado como un estudio sobre "las actitudes de estudiantes universitarios hacia varios grupos sociales en el contexto costarricense".

Antes de iniciar, las personas participantes escucharon los lineamientos de participación y el consentimiento informado, el cual se elaboró siguiendo las directrices del Comité Ético-Científico de la Universidad de Costa Rica. Este incluía los aspectos de riesgos, beneficios, y la garantía de participación anónima y confidencial. Asimismo, se enfatizó en que la participación era voluntaria, por lo que la persona se podía negar a participar o incluso interrumpir su participación sin que esto le perjudicara. Todas las personas accedieron a participar, luego de lo cual se les hizo entrega de los cuestionarios.

Las personas de cada grupo de clase fueron asignadas aleatoriamente a una de las dos condiciones, ya sea de control o experimental, con el fin de establecer grupos estadísticamente comparables y eliminar o disminuir los efectos de variables extrañas tales como la capacidad olfativa, las actitudes previas hacia las personas homosexuales, etc. 
Las personas asignadas a la condición experimental fueron conducidas a otra aula del mismo edificio, en la cual se había dispersado el olor desagradable, y contestaron el cuestionario al mismo tiempo que el grupo control. Una vez que todas las personas participantes entregaron el cuestionario respondido, se les explicó en qué consistía el experimento, incluyendo la definición de embodied cognition y la hipótesis del estudio. Además se verificó que cada persona se encontrara bien al finalizar su participación.

\section{Análisis}

La verificación de la manipulación y el encubrimiento de la hipótesis fueron explorados mediante distribuciones de frecuencias. Se utilizó la prueba de Kolmogorov-Smirnov ( $p$ < .001) para examinar que las distribuciones de las puntuaciones tanto en el termómetro de sentimientos como en la escala ATLG no difirieran significativamente de la distribución normal. Las diferencias entre el grupo experimental y el grupo control fueron calculadas mediante la prueba t de Student para muestras independientes. El tamaño del efecto fue estimado mediante el cálculo de la d de Cohen y sus índices fueron interpretados como grandes $(<.8)$, medianos $(<.5)$ y pequeños $(<.2)$ según la definición convencional de Cohen (1988). Los análisis fueron Ilevados a cabo mediante el programa IBM SPSS Statistics (v. 20) para Mac OS.

\section{Resultados}

Previo a los análisis principales, se exploraron los resultados de las preguntas destinadas a la verificación de la manipulación. Todas las personas del grupo experimental reportaron no presentar condiciones que pudieran disminuir su capacidad olfativa, aunque cuatro de ellas (17.4\%) indicaron haber sufrido de resfriado en los últimos días. Asimismo, al preguntar de manera abierta si hubo algún factor ambiental que hubiera intervenido al momento de contestar las preguntas, doce personas del grupo experimental (52.2\%) mencionaron el olor de manera espontánea.

Por otra parte, respecto al encubrimiento de la hipótesis, aunque seis personas del grupo experimental (26.1\%) respondieron sí haber sospechado que el olor era parte del experimento (cuando se les preguntó directamente al final del cuestionario), ninguna persona de la muestra total mencionó esta variable cuando se les solicitó anotar cuál consideraban que era la hipótesis que se sometía a prueba con el estudio.

Respecto a la prueba de hipótesis, en la Tabla 1 se presentan los resultados obtenidos mediante el termómetro de sentimientos. Como se puede observar, las personas expuestas al olor desagradable indicaron actitudes más desfavorables 
hacia todos los grupos sociales, aunque no todas estas diferencias fueron estadísticamente significativas. Acorde con la hipótesis de nuestra investigación, el efecto se dio con mayor intensidad en el caso de las actitudes hacia los hombres homosexuales, en donde se encontró que las personas que fueron expuestas al olor desagradable presentaron actitudes más negativas hacia este grupo social $(\mathrm{M}=68.26, \mathrm{DE}=20.43)$ que las personas del grupo control $(\mathrm{M}=$ 87.78, $\mathrm{DE}=18.33)$. Esta diferencia principal fue significativa $\left(\mathrm{t}_{(39)}=3.17, \mathrm{p}=\right.$ .003) y el tamaño del efecto fue muy grande $(d=1.01)$, superando las diferencias encontradas en las actitudes hacia los restantes grupos sociales. Además de este hallazgo, se encontraron otras diferencias significativas con un tamaño de efecto grande en las actitudes hacia los grupos de personas afrocostarricenses $(p=.003, d=0.98)$, hacia mujeres tanto heterosexuales $(p=.007, d=0.91)$ como homosexuales ( $p=.008, d=0.88)$, y hacia las personas nicaragüenses ( $p$ $=.012, \mathrm{~d}=0.82$ ), así como un efecto de tamaño medio en las actitudes hacia personas campesinas ( $p=.024, d=0.74$ ).

Finalmente, en la Tabla 2 se muestra los resultados obtenidos con la escala ATLG, destinada a estimar específicamente las actitudes hacia personas homosexuales, tanto hacia lesbianas (ATL) como hacia gays (ATG). Como se puede observar, también con esta escala se encontró que las personas expuestas al olor desagradable presentaron actitudes más negativas hacia las personas homosexuales $(\mathrm{M}=1.67, \mathrm{DE}=0.62$ ) que las personas del grupo control ( $\mathrm{M}$ $=1.47, \mathrm{DE}=0.43)$, sin embargo estas diferencias no fueron estadísticamente significativas $\left(\mathrm{t}_{(39)}=1.21, \mathrm{p}=.23\right)$, y de asumirse diferencias el tamaño del efecto sería pequeño $(\mathrm{d}=0.37)$. Aun así, la probabilidad de error de que estas diferencias se deban al azar es mayor en el caso de las actitudes hacia las lesbianas (51\%) que en el caso de las actitudes hacia los hombres homosexuales (13\%).

Tabla 1

Actitudes hacia grupos sociales según condición experimental

\begin{tabular}{lllllllll}
\hline & \multicolumn{7}{c}{ Grupo control } & \multicolumn{2}{l}{$\begin{array}{l}\text { Grupo } \\
\text { experimental }\end{array}$} \\
\cline { 3 - 5 } Grupo & $\mathrm{M}$ & $\mathrm{DE}$ & $\mathrm{M}$ & $\mathrm{DE}$ & $\mathrm{t}(39)$ & $\mathrm{p}$ & $\mathrm{d}$ de Cohen \\
\hline P indígenas & 75.00 & 29.31 & 65.43 & 19.48 & 1.19 & .24 & 0.38 \\
H heterosexuales & 91.11 & 16.23 & 80.87 & 21.83 & 1.72 & .09 & 0.53 \\
P chinas & 71.11 & 24.41 & 58.91 & 17.51 & 1.79 & .08 & 0.57 \\
P colombianas & 75.83 & 23.59 & 63.91 & 19.60 & 1.77 & .08 & 0.55
\end{tabular}




$\begin{array}{lccccccc}\text { P ateas } & 71.94 & 25.56 & 59.35 & 24.23 & 1.61 & .12 & 0.51 \\ \text { P obesas } & 65.56 & 27.43 & 56.09 & 22.46 & 1.22 & .23 & 0.38 \\ \text { H homosexuales } & 87.78 & 18.33 & 68.26 & 20.43 & 3.17 & .003 & 1.01 \\ \text { P pobres } & 76.67 & 32.13 & 60.65 & 23.47 & 1.84 & .07 & 0.57 \\ \text { M homosexuales } & 82.78 & 23.47 & 62.39 & 23.15 & 2.78 & .008 & 0.88 \\ \text { P campesinas } & 84.44 & 20.28 & 70.00 & 18.89 & 2.35 & .024 & 0.74 \\ \text { P adultas mayores } & 85.83 & 18.65 & 75.65 & 23.08 & 1.52 & .14 & 0.48 \\ \text { P católicas } & 62.50 & 28.50 & 58.70 & 23.80 & 0.47 & .64 & 0.14 \\ \text { M heterosexuales } & 89.44 & 16.79 & 73.04 & 19.23 & 2.86 & .007 & 0.91 \\ \text { P nicaragüenses } & 76.94 & 22.50 & 59.13 & 20.87 & 2.62 & .012 & 0.82 \\ \text { P afrocostarricenses } & 85.56 & 20.50 & 66.09 & 19.13 & 3.13 & .003 & 0.98\end{array}$

Nota: $\mathrm{P}=$ Persona; $\mathrm{H}=$ Hombre; $\mathrm{M}=$ Mujer.

Tabla 2

Actitudes hacia personas homosexuales según condición experimental

\begin{tabular}{lccccccc}
\hline \multicolumn{7}{c}{ Grupo control } & \multicolumn{2}{c}{ Grupo experimental } \\
Escala & $\mathrm{M}$ & $\mathrm{DE}$ & $\mathrm{M}$ & $\mathrm{DE}$ & $\mathrm{t}(39)$ & $\mathrm{p}$ & $\mathrm{d}$ de Cohen \\
\hline ATL & 1.43 & 0.47 & 1.54 & 0.58 & 0.66 & .51 & 0.21 \\
\hline ATG & 1.52 & 0.49 & 1.81 & 0.69 & 1.55 & .13 & 0.48 \\
\hline ATLG & 1.47 & 0.43 & 1.67 & 0.62 & 1.21 & .23 & 0.37 \\
\hline
\end{tabular}

Nota: $A T L=$ Escala de actitudes hacia lesbianas (10 ítems); ATG = Escala de actitudes hacia gays (10 ítems); M = Escala de actitudes hacia personas homosexuales (20 ítems).

De manera adicional, con base en los hallazgos previos de Moral (2013) y Smith-Castro y Molina-Delgado (2011), se analizaron las diferencias entre hombres y mujeres en cuanto a sus actitudes hacia las personas homosexuales (Tabla 3 ). 
Estos análisis resultan exploratorios en vista de lo reducido de la muestra y la diferencia en la cantidad de participantes hombres $(n=8)$ y mujeres $(n=33)$. En términos generales, se observa que los hombres presentaron actitudes más negativas hacia las personas homosexuales que las mujeres, reflejadas en una menor calidez en el termómetro de sentimientos y un mayor prejuicio en la escala ATLG. Sin embargo, estas diferencias no fueron significativas.

Tabla 3

Actitudes hacia personas homosexuales según sexo

\begin{tabular}{|c|c|c|c|c|c|c|c|}
\hline \multirow[b]{2}{*}{ Grupo / Escala } & \multicolumn{2}{|c|}{ Mujeres } & \multicolumn{2}{|c|}{ Hombres } & \multirow[b]{2}{*}{$\mathrm{t}(39)$} & \multirow[b]{2}{*}{$\mathrm{p}$} & \multirow[b]{2}{*}{ d de Cohen } \\
\hline & $M$ & DE & $M$ & DE & & & \\
\hline H homosexuales & 77.27 & 21.29 & 75.00 & 24.50 & 0.26 & .79 & 0.10 \\
\hline M homosexuales & 70.76 & 25.80 & 73.75 & 23.87 & 0.30 & .77 & 0.12 \\
\hline ATL & 1.45 & 0.47 & 1.64 & 0.74 & 0.88 & .40 & 0.31 \\
\hline ATG & 1.63 & 0.55 & 1.91 & 0.87 & 0.67 & .52 & 0.38 \\
\hline ATLG & 1.54 & 0.47 & 1.77 & 0.80 & 0.79 & .45 & 0.35 \\
\hline
\end{tabular}

\section{Comentarios}

En la presente investigación se sometió a prueba una hipótesis específica de las teorías de cognición corporalizada. Estas teorías plantean que los procesos cognitivos se ven influenciados por los estados internos del cuerpo, así como por sus movimientos (Lakoff \& Johnson, 1999). Entre estos procesos cognitivos se encuentran las actitudes hacia grupos sociales, con lo que se plantea que las actitudes se ven influenciadas no solo por las creencias personales, el aprendizaje y el contexto social, sino además por factores relativos al organismo biológico que sustenta los procesos cognitivos. En este sentido, se buscó someter a prueba la hipótesis específica derivada del estudio de Inbar et al. (2012), quienes encontraron que la inducción experimental de asco generaba actitudes más negativas hacia los hombres homosexuales.

En relación con el aspecto más general de la cognición corporalizada, los hallazgos de nuestra investigación indican un efecto estadísticamente significativo del estado emocional de asco sobre las actitudes hacia seis de los 15 grupos sociales evaluados. Este resultado evidencia claramente un ejemplo de cognición 
corporalizada, pues el estado del cuerpo característico de la emoción de asco demostró tener influencia sobre variables cognitivas complejas como lo son las actitudes sociales. En este estudio se observó cómo al someter a las personas participantes a un olor desagradable, la reacción fisiológica propia del asco afectó las cogniciones de los participantes de manera que reportaron menores niveles de actitudes positivas (calidez) hacia hombres homosexuales, personas afrocostarricenses, mujeres heterosexuales, mujeres homosexuales, personas nicaragüenses y personas campesinas.

Por otra parte, otros de nuestros hallazgos coinciden plenamente con los resultados encontrados por Inbar et al. (2012), a saber, las actitudes hacia hombres homosexuales fueron las mayormente influenciadas luego de la manipulación experimental del asco. Si bien las actitudes hacia otros cuatro grupos sociales también fueron influenciadas de manera muy significativa debido a la exposición al olor desagradable, consideramos importante hacer notar que el efecto del asco sobre las actitudes hacia las mujeres no fue específico hacia las lesbianas, sino que se presentó tanto hacia mujeres homosexuales como heterosexuales. En cambio, el efecto del asco sobre las actitudes hacia los hombres sí fue específico hacia los hombres homosexuales.

Los resultados obtenidos al utilizar una medición más compleja de las actitudes hacia las personas homosexuales mediante la escala Attitudes Toward Lesbians and Gay Men (ATLG) no resultaron estadísticamente significativos. Sin embargo, una vez más, la tendencia a nivel de promedios mostró un mayor efecto del asco sobre las actitudes hacia los gays $(d=0.48)$ que sobre las actitudes hacia las lesbianas $(d=0.21)$, mientras que la probabilidad de error de estos hallazgos fue del $13 \%$ en el caso de las actitudes hacia los gays, en contraste con el $51 \%$ de probabilidad de error en el caso de las actitudes hacia las lesbianas.

De manera complementaria, se analizaron las diferencias entre hombres y mujeres en cuanto a sus actitudes hacia personas homosexuales. Se observó que los hombres presentaron actitudes más negativas hacia las personas homosexuales que las mujeres, reflejadas en una menor calidez en el termómetro de sentimientos y un mayor prejuicio en la escala ATLG. Este hallazgo fue exploratorio, en vista del tamaño de la muestra y de la falta de significancia estadística. Sin embargo coincide con los resultados de Moral (2013) y SmithCastro y Molina-Delgado (2011), quienes observaron que los hombres presentaron actitudes más negativas hacia las personas homosexuales que las mujeres. Una conclusión similar fue encontrada por Herek y González-Rivera (2006), quienes obtuvieron el mismo resultado en una muestra de 616 hijos de inmigrantes mexicanos en los Estados Unidos.

En conclusión, el estudio aporta evidencia en el contexto costarricense de fenómenos derivados de las teorías de cognición corporalizada. Sin embargo, las evidencias de un efecto del correlato fisiológico del asco sobre las actitudes hacia 
específicamente los hombres homosexuales no fueron consistentes. Se considera que el tamaño reducido de la muestra pudo contribuir a dicha inconsistencia, por lo que resulta necesaria mayor investigación al respecto.

\section{Notas}

1 Esta investigación se llevó a cabo como parte del curso Procesos Psicológicos Básicos, durante el II ciclo del año 2013.

Traducción revisada por Licda. Rocío Razos, Licenciada en Enseñanza del Inglés y en Administración Educativa, además de Bachiller en Inglés.

\section{Bibliografía}

Balluerka, N. \& Vergara, A. I. (2002). Diseños de investigación experimental en psicología: Modelos y análisis de datos mediante el SPSS 10.0. Madrid, España: Pearson.

Barrientos, J. E. \& Cárdenas, M. (2012). A confirmatory factor analysis of the spanish language version of the Attitudes Toward Lesbians and Gay Men Scale (ATLG). Universitas Psychologica, 11(2), 579-586.

Bem, D. (1972). Self-perception theory. En L. Berkowitz (Ed.), Advances in experimental social psychology (Vol. 6, pp. 1-62). Nueva York, Estados Unidos: Academic Press.

Cárdenas, M. \& Barrientos, J. (2008). The Attitudes toward Lesbians and Gay Men Scale (ATLG): Adaptation and testing the reliability and validity in Chile. Journal of Sex Research, 45(2), 140-149.

Cohen, J. (1988). Statistical power analysis for the behavioral sciences (2a. ed.). Nueva York, Estados Unidos: Academic Press.

Cuddy, A. J. C., Fiske, S. T., Kwan, V. S. Y., Glick, P., Demoulin, S., Leyens, J.-P., Ziegler, R. (2009). Stereotype content model across cultures: Towards universal similarities and some differences. British Journal of Social Psychology, 48, 1-33.

Durante, F., Fiske, S. T., Kervyn, N., Cuddy, A. J. C., Akande, A., Adetoun, B. E. ... Storari, C. C. (2013). Nations' income inequality predicts ambivalence in stereotype content: How societies mind the gap. British Journal of Social Psychology, 52(4), 726-746. 
Fournier, M. V. (2008). Evaluación de actitudes discriminativas y prejuicios hacia diversas poblaciones, con estudiantes de universitarios de la gran área metropolitana de San josé, Costa Rica. San José, Costa Rica: Centro de Investigación y Promoción para América Central de Derechos Humanos.

Herek, G. M. (1988). Heterosexuals' attitudes toward lesbians and gay men: Correlates and gender differences. Journal of Sex Research, 25, 451-477.

Herek, G., \& González Rivera, M. (2006). Attitudes toward homosexuals among U.S. residents of Mexican descendent. Journal of Sex Research, 43(2), 1-25.

Hernández, R., Fernández, C. \& Baptista, P. (2010). Metodología de investigación (5a. ed.). México D.F.: McGraw-Hill.

Inbar, Y., Pizarro, D. A. \& Bloom, P. (2012). Disgusting smells cause decreased liking of gay men. Emotion, 12(1), 23-27.

Laird, J. D. (1974). Self-attribution of emotion: The effects of expressive behavior on the quality of emotional experience. Journal of Personality and Social Psychology, 29(4), 475-486.

Lakoff, G. \& Johnson, M. (1999). Philosophy in the flesh: The embodied mind and its challenge to Western thought. Nueva York, Estados Unidos: Basic Books.

Leung, A., Qiu, L., Ong, L. \& Tam, K. (2011). Embodied cultural cognition: Situating the study of embodied cognition in socio-cultural contexts. Social \& Personality Psychology Compass, 5(9), 591-608.

Meier, B. P., Schnall, S., Schwarz, N. \& Bargh, J. A. (2012). Embodiment in social psychology. Topics in Cognitive Science, 4(4), 705-716.

Moral, J. (2013). Homofobia, religión e ideología política en la Encuesta Nacional de Cultura Política y prácticas ciudadanas (ENCUP2008). Estudios sobre las Culturas Contemporáneas, 19(37), 99-128.

Schnall, S., Haidt, J., Clore, G. L., \& Jordan, A. H. (2008). Disgust as embodied moral judgment. Personality and Social Psychology Bulletin, 34(8), 10961109.

Shadish, W., Cook, T. \& Campbell, D. (2002). Experimental and quasi-experimental designs for generalized causal inference. Boston, Estados Unidos: Houghton Mifflin Company. 
Smith-Castro, V. \& Molina-Delgado, M. (2011). Actitudes hacia el matrimonio y la unión civil gay en Costa Rica: ¿religiosidad, homofobia, autoritarismo o desconocimiento? Interamerican Journal of Psychology, 45(2), 133-143.

Solarz, A. (1960). Latency of instrumental responses as a function of compatibility with the meaning of eliciting verbal signs. Journal of Experimental Psychology, $59,239-245$.

Suárez, D. \& Madrigal, F. (2007). Percepción de estudiantes universitarios sobre la homo/lesbofobia en Costa Rica. San José, Costa Rica: Centro de Investigación y Promoción para América Central de Derechos Humanos.

Wells, G. L. \& Petty, R. E. (1980). The effects of overt head movements on persuasion: Compatibility and incompatibility of responses. Basic and Applied Social Psychology, 1, 219-230.

\section{Anexo}

\section{Protocolo de consentimiento informado oral}

En este proyecto de investigación se busca estimar las actitudes de estudiantes universitarios hacia varios grupos sociales en el contexto costarricense. Se espera así obtener información que permita aclarar aspectos relacionados con la tendencia costarricense de contemplar cada uno de los temas o las diferencias entre grupos sociales.

Qué se hará: Se solicitará a los y las participantes llenar un cuestionario. La duración total del proceso será de aproximadamente diez minutos.

Riesgos: Esta actividad no presenta riesgos de ningún tipo (psicológico, físico o legal). Algunas preguntas podrían resultarle incómodas o repetitivas, por lo que si no se siente cómodo(a) en algún momento, podrá comunicarle a las personas investigadoras que no desea seguir participando en el proyecto, sin que esto tenga ninguna consecuencia negativa para usted.

Beneficios: Con la participación en el proyecto no se obtendrá ningún beneficio directo, sin embargo es posible que las investigadoras, puedan generar mayores conocimientos acerca de las manifestaciones y actitudes cognoscitivas de las personas así como un mayor aprendizaje acerca de la opinión de la juventud actual.

Voluntariedad: Su participación en la investigación es voluntaria, por lo que tienen derecho a participar, negarse o interrumpir su participación en cualquier momento, sin que esto le perjudique. 
Confidencialidad: Su participación en este proyecto es confidencial. Los resultados serán utilizados para elaborar los resultados de la investigación sin ningún nombre y únicamente podrían ser divulgados en publicaciones o reuniones científicas de manera anónima.

Información: Si desea más información después de haber participado en el proyecto de investigación, podrá comunicarse con los teléfonos que vamos a poner en la pizarra.

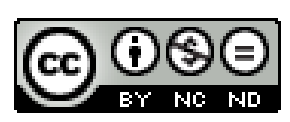

Este obra está bajo una licencia de Creative Commons ReconocimientoNoComercial-SinObraDerivada 4.0 Internacional. 